\title{
TV/Series
}

12 | 2017

Littérature et séries télévisées/Literature and TV series

\section{Les fonctions de l'écrit intime dans la constitution du héros : Jackson Teller, volonté intime et destin tragique}

\section{Vanessa Loubet-Poëtte}

\section{OpenEdition}

\section{Journals}

Édition électronique

URL : http://journals.openedition.org/tvseries/2175

DOI : 10.4000/tvseries. 2175

ISSN : 2266-0909

Éditeur

GRIC - Groupe de recherche Identités et Cultures

Référence électronique

Vanessa Loubet-Poëtte, « Les fonctions de l'écrit intime dans la constitution du héros : Jackson Teller, volonté intime et destin tragique », TV/Series [En ligne], 12 | 2017, mis en ligne le 20 septembre 2017, consulté le 01 mai 2019. URL : http://journals.openedition.org/tvseries/2175 ; DOI : 10.4000/ tvseries. 2175

Ce document a été généré automatiquement le 1 mai 2019.

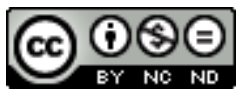

TV/Series est mis à disposition selon les termes de la licence Creative Commons Attribution - Pas d'Utilisation Commerciale - Pas de Modification 4.0 International. 


\title{
Les fonctions de l'écrit intime dans la constitution du héros : Jackson Teller, volonté intime et destin tragique
}

\author{
Vanessa Loubet-Poëtte
}

1 La série télévisée Sons of Anarchy, diffusée de 2008 à 2014, sur la chaîne FX, décrit le fonctionnement d'une microsociété anarchique qui refuse l'ordre établi mais qui fixe sans cesse ses propres règles de vie. Même s'il a partagé le quotidien d'un véritable motorcycle club de Californie lors de la préparation de la série, son créateur, Kurt Sutter, n'entend pas faire œuvre documentaire, à la manière de David Simon pour The Wire par exemple ${ }^{1}$. Bien au contraire, les velléités réalistes cèdent vite la place à un resserrement sur les relations familiales au sein du clan des Teller-Morrow, et à une mise en lumière épique et tragique des événements.

2 Comme l'explique Sylvaine Bataille en analysant autant les références de la série que la promotion faite par la chaîne FX pour les trois premières saisons, Sons of Anarchy apparaît comme une relecture d'Hamlet, transportant la cour d'Elseneur sous le soleil cuisant de Charming en Californie ${ }^{2}$. Des premiers slogans («Hamlet on Harleys", « Hamlet in black leather ${ }^{3}$ ) jusqu'aux images promotionnelles pour le lancement de la $7^{\mathrm{e}}$ et dernière saison (Fig. 1) dans lesquelles un crâne très shakespearien apparaît sur le dos du héros, la filiation au Prince du Danemark est affichée. Point d'orgue, l'épigraphe final ${ }^{4}$ cite le texte au sein de la série et parachève l'appropriation de la pièce. Car il est bien question dans Sons of Anarchy du fantôme d'un père, John Thomas ou J.T., et de son fils, Jackson ou Jax, et de la "folie» potentielle de ce dernier, face au destin profilé devant lui. Mais, différence essentielle dans le cadre de cette réflexion sur les écrits intimes, ce dialogue ne passe pas par le truchement d'un spectre théâtral comme sur la scène du théâtre élisabéthain, mais par l'entremise de différents écrits laissés, hérités, trouvés, volés, cachés, piégés... Des écrits assumant un rôle actanciel presque aussi intense que ceux des personnages de chair et de sang. 
Fig. 1 : Image promotionnelle pour le lancement de la $7^{\mathrm{e}}$ saison.

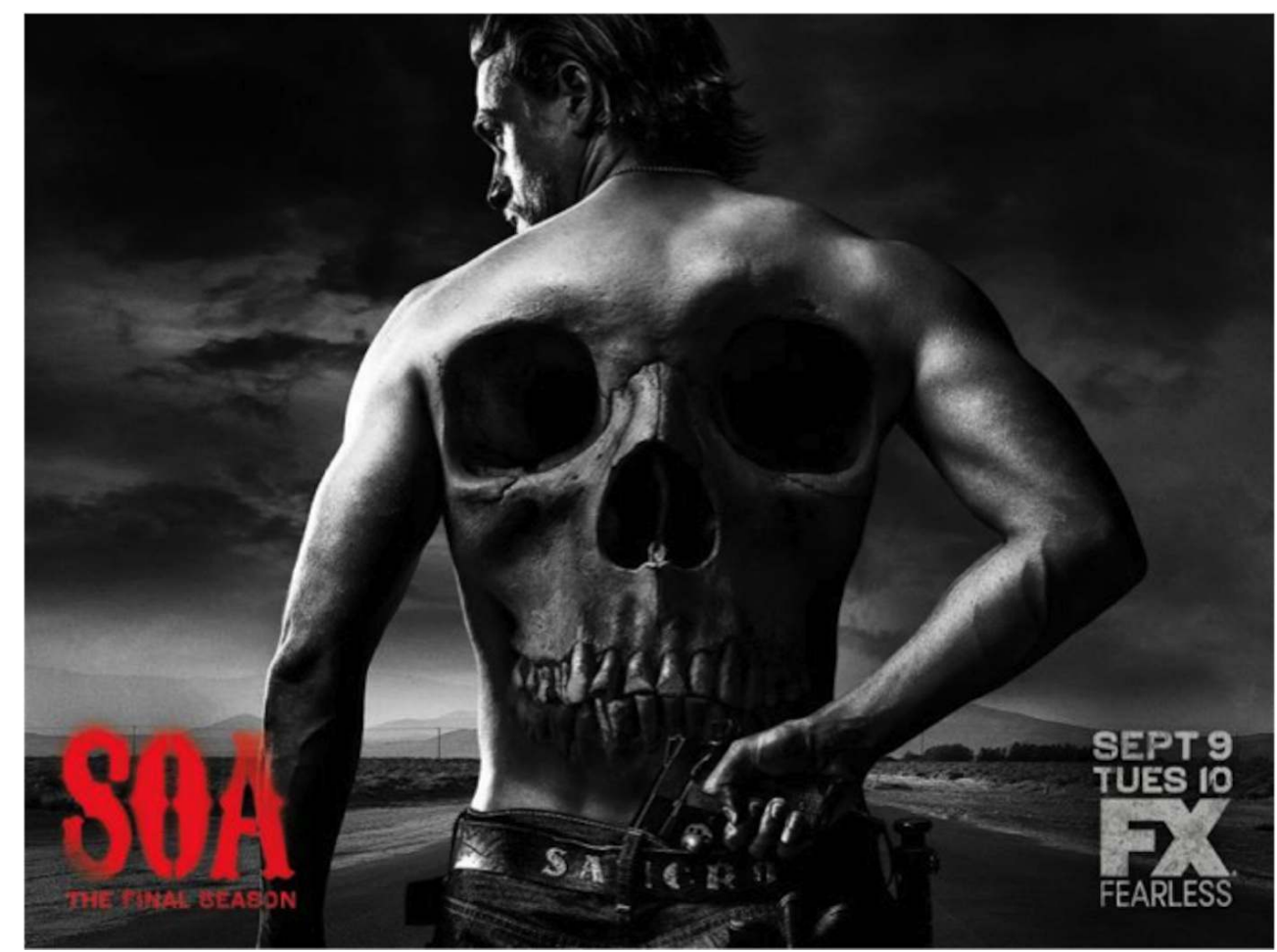

Cette étude attire l'attention sur trois types d'écrits intimes. Il y a tout d'abord le manuscrit intitulé The Life and Death of Sam Crow - How the sons of anarchy lost their way, signé par J.T. Teller. Le père décédé, un des neufs membres fondateurs du SAMCRO (Sons of Anarchy Motorcycle Club Redwood Original), y a consigné ses ambitions progressistes et sa volonté de faire évoluer vers une voie légale son organisation qui vit du trafic d'armes. Ce sont aussi ses lettres écrites à Maureen Ashby, sa maîtresse en Irlande, avec laquelle il a eu une fille nommée Trinity ${ }^{5}$. Et ce sont enfin les carnets noircis par son fils Jax, à partir de son séjour en prison entre la $3^{\mathrm{e}}$ saison et la $4^{\mathrm{e}}$ saison, où le héros s'adonne à des réflexions semblables à celles de son père sur son destin individuel et sur celui du club. Mentionnons aussi un autre journal au début de la $6^{\mathrm{e}}$ saison, tenu par un jeune garçon coupable d'une tuerie dans son école, dont la représentation est associée à celui de Jax, augure d'un mauvais présage et précipitation vers l'issue finale ${ }^{6}$. Tous ces textes présentent plusieurs points communs, à commencer par leur incomplétude. Les téléspectateurs n'y ont accès que partiellement, dans des circonstances particulières, lorsqu'ils sont lus dans la diégèse, le plus souvent en voix off. Fragmentaires par essence, ils conviennent au format de la série. De plus, ces textes sont tabous : leur lecture est interdite tant ils sont lourds de secrets. Ils ont également tous une valeur programmatique puisqu'ils excèdent largement le champ de la confidence pour mener des réflexions. Enfin, ce sont des textes «intimes », dans tous les sens du terme. Intimes d'abord parce qu'ils accèdent à ce qu'il y a de plus profond, à ce qui définit leur auteur. Moyen de se connaître et de se construire, l'écrit intime est plus ou moins consciemment inscrit dans une visée cathartique, puisque «le journal intime est toujours l'écho d'un malaise plus ou moins profond de l'individu en face de lui-même, des autres et de l'existence ", comme l'explique Alain Girard'. Dans Sons of Anarchy, le père comme le fils écrivent parce qu'ils doutent, ou plutôt parce qu'ils ne veulent plus douter. Intimes aussi 
parce qu'ils façonnent un espace du privé et du secret, auquel il convient d'autoriser ou non l'accès aux potentiels lecteurs. Le caché et le révélé sont alors sans cesse agencés en fonction des relations actancielles. Et pourtant, le journal intime s'avère le moins bon moyen de conserver des secrets. Philippe Lejeune et Catherine Bogaert remarquent que « l'intimité complète ne s'obtiendrait peut-être... qu'en renonçant au journal! » puisque «le véritable secret ne s'obtient [...] que par l'auto-censure ${ }^{8}$.» Conseil suivi par Jackson, qui détruit tous les textes, à la fin de la série.

Afin de mesurer ce que la série Sons of Anarchy emprunte à la littérature pour sa construction narrative et pour son usage de l'écrit intime, nous nous proposons d'interroger la littérarité de ces textes et d'analyser leurs échos entre tragique et intime à l'œuvre dans la construction psychologique, actancielle et ontologique du personnage de Jackson Teller9.

\section{Readers, writers... and bikers?}

Pour aussi surprenant que cela puisse paraitre, il y a dans Sons of Anarchy presque autant de livres que de motos ; à l'image de leur créateur qui arbore un style vestimentaire et des tatouages évoquant dans l'imaginaire collectif plutôt un homme de peu de foi qu'un homme de lettres, les bikers de Charming sont aussi des lecteurs et des scripteurs.

\section{Textes et écrits}

6 Plusieurs types de livres sont lus et échangés, que ce soit en prison où ils servent de passe-temps ou de caches pour des messages et des armes ${ }^{10}$, ou dans la sphère familiale où Gemma fait la lecture à son petit-fils Abel. Signalons la présence de la Bible, bien que plutôt bafouée lorsqu'elle permet de dissimuler une seringue destinée à tenter une toxicomane ${ }^{11}$. Certains titres notables offrent des pistes de lecture pour la situation diégétique. The Jungle ${ }^{12}$, description de l'abomination des conditions de vie et de travail des employés immigrés des abattoirs de Chicago, peut évoquer les multiples carnages perpétrés et l'affrontement des gangs ethniques. The Mad King ${ }^{13}$ est une référence à la romance ruritanienne : ce genre populaire né à la fin du xix siècle, mêlant action et amour au sein de petites monarchies européennes, n'est pas sans rappeler le microcosme de Charming. Le poème "My comforter ${ }^{14}$ ", extrait d'une anthologie des sœurs Brontë, résonne lors de moments d'intimité entre deux détenus et suggère, en dépit de sa visée première, une extrême violence, puisque leur relation est forcée et placée sous le sceau de la vengeance. Enfin, le personnage de Piney Winston semble avoir succombé à l'effet de mode de la littérature zombie quand il lit Cycle Zombie signé par Stephen King ${ }^{15}$. N'en déplaise aux amateurs du genre, ce livre dont la couverture laisse supposer une histoire aux confins des univers de Rick Grimes ${ }^{16}$ et de Jackson Teller est une pure création de la série, résultant d'une proposition non sans humour du célèbre auteur américain, qui fait une apparition lors de la même saison, sous les traits d'un nettoyeur nommé Bachman, en hommage à un autre de ses noms de plume ${ }^{17}$. Dernière lecture d'un homme sur le point d'être froidement exécuté, la référence n'en est que plus délectable. Clins d'œil aux téléspectateurs avisés, capables d'en saisir la pertinence ou l'ironie, ces mentions jalonnent l'intrigue autour des journaux intimes. À cet égard, un échange entre deux personnages fait pleinement sens. À Gemma qui lui demande si le carton trop lourd pour 
elle contient des armes ou des munitions, Nero répond «Books», qualifiés en retour par celle qui ne cesse de nouer les ficelles du secret de « way more dangerous $»^{18}$.

Autre forme d'écrit plus coutumière des bikers, les tatouages ont plusieurs fonctions, suggérées toutes ensemble dans le générique : ils signalent l'appartenance à la "tribu ", par les codes iconographiques qui lui sont propres (la Faucheuse sur tous les membres du club), ils affichent et subliment des repères généalogiques (la tombe de J.T. ou les prénoms de ses fils sur Jax) et ils affirment une identification individuelle, faisant de chaque tatoué une entité singulière (les tatouages en miroir de Juice qui incarne la figure duelle du traitre ou les smileys d'Happy décomptant le nombre de ses victimes) ${ }^{19}$.

Moins glorieuse dans ce monde de hors-la-loi, la confession destinée aux autorités constitue une dernière forme d'écrit, qui concerne presque tous les personnages, qu'ils y soient contraints ou non. Coopération entre ceux qui tentent de faire respecter la loi et ceux qui l'enfreignent, s'il accède à une valeur juridique, l'aveu écrit est toujours synonyme de traitrise et avilit les deux parties ${ }^{20}$.

9 Malgré leur récurrence, difficile de dire que les livres et les écrits trouvent de grands lecteurs, auxquels ils offriraient des solutions, des retraites ou des points de repères éthiques. En témoigne cette scène entre Jax et Wendy lors des premiers jours de vie de leur fils Abel. À Wendy qui remarque ne pas trouver un chapitre à l'attention des jeunes mères ex-junkies dans un manuel pour parents novices, Jax répond: "I don't think our pages have been written yet, sweetheart ${ }^{21}$.». C'est juste, compte tenu du haut degré de marginalité de tous les personnages et de la complexité des intrigues ; mais c'est faux, du fait des questions morales centrales - ne serait-ce que celle du devoir - qui ne diffèrent pas de celles des grandes tragédies ou des grands épisodes de la mythologie. C'est peutêtre parce qu'ils n'identifient pas ces références extradiégétiques que les personnages font preuve d'une si forte propension à vouloir caractériser les écrits diégétiques, c'est-àdire les textes intimes. Citons ces quelques réactions: Chibs qui plaisante sur le fait que Jax écrit un livre pour enfants, quand il s'agit d'un livre pour ses enfants ${ }^{22}$; Clay, figure paternelle négative, et donc peu sensible et enclin à l'écriture, qui qualifie les textes de " bunch of words [...], shit ${ }^{23} "$, "bunch of love letters, some half-gay poems and shit $\left.{ }^{24} »\right)$, masse de mots qu'il ne sert à rien d'analyser ; Tara qui ironise sur le fait qu'un biker puisse tenir un journal intime et verbalise l'incrédulité même des téléspectateurs face à la situation et à la série ("My badass biker is journaling ${ }^{25}$ ?»). Si elles peuvent être identifiées comme marques d'humour, ces répliques sont surtout, sans nul doute, l'occasion pour Kurt Sutter de s'amuser des porosités entre les domaines du fictif, celui de la littérature qui existe indépendamment de l'univers de Sons of Anarchy et celui délimité par la série.

\section{Mécanique scénaristique}

Plus que de simples échos à la littérature ou à l'intériorité, ces écrits intimes intègrent et alimentent la mécanique scénaristique de la série. À défaut d'un inventaire exhaustif, le tableau en annexe récapitule la place et la récurrence des moments consacrés à l'écrit intime tout au long des sept saisons, avec deux usages, selon que le texte est directement cité ou seulement évoqué. Plusieurs remarques émanent à l'observation de ces correspondances. On perçoit tout d'abord la relative succession des trois ensembles de textes, comme ressorts narratifs distincts : le manuscrit de J.T. est opérant surtout dans la $1^{\text {re }}$ saison, les lettres adultérines ne le sont que dans la $4^{\mathrm{e}}$. Une fois l'arc narratif épuisé, les secrets et les vérités compromettantes révélés, le texte est mis de côté. De plus, en 
ouverture ou en fermeture de saison, la convocation d'un ou de plusieurs textes intimes crée des nœuds narratifs efficaces, tant pour clore ou lancer des intrigues de longue haleine que pour associer plusieurs voix. Fréquemment, les scènes de lecture ou d'écriture sont situées en début d'épisode coïncidant avec le réveil des personnages, soit un moment d'intimité et de répit avant les péripéties de la journée. De plus, la présence importante du manuscrit de J.T. dans la $1^{\text {re }}$ saison et sa mise en retrait progressive est pleinement symptomatique de l'évolution du héros : agissant au départ, tel un tout jeune adulte, avec ou contre les conseils qu'il peut lire de son père, Jax s'en affranchit, jusqu'à rédiger son propre journal, à partir de la $4^{\mathrm{e}}$ saison. De même, la disparition ou presque de tout texte intime dans l'ultime saison indique que, s'il y avait jusque-là un temps de l'écrit parallèle au temps de l'action, il ne reste désormais de place que pour l'action : le repli sur soi et la trêve n'ont plus cours dans le déferlement de violence et de vengeance des derniers instants. Toutefois, il faut repérer la présence des deux journaux dans l'épisode final, apparaissant pour mieux disparaître à jamais par le feu, clôturant ainsi le cycle des générations et par la même occasion celui de la série.

\section{3. Écrivant et écrivain}

11 À première vue, Jax Teller et son père incarnent la figure de l'intimiste-écrivant, c'est-àdire, en suivant la définition de Roland Barthes, celui qui utilise l'écrit avant tout comme un moyen d'extérioriser sa pensée, de "supporte[r] un faire ", sans ambition stylistique, réflexive ou littéraire ${ }^{26}$. Pourtant, ils manifestent tous deux un soin particulier à l'écriture, ritualisée et même sacralisée, plusieurs personnages ne manquant pas d'évoquer le fait que le père était doué et de supposer que ce don, parmi d'autres, est héréditaire. D'autant plus que le caractère fragmentaire, induit par la tenue du journal intime et par ses modes de citation dans la série, tend à élever ces écrits au rang de formules rhétoriques et éloquentes.

12 Un autre personnage questionne ce rapport au texte, au geste d'écriture et à la figure de l'écrivain, il s'agit d'Otto Delaney (Fig. 2).

Fig. 2 : Otto Delaney / Kurt Sutter, scripteur / scénariste (« Wolfsangel », 6.04).

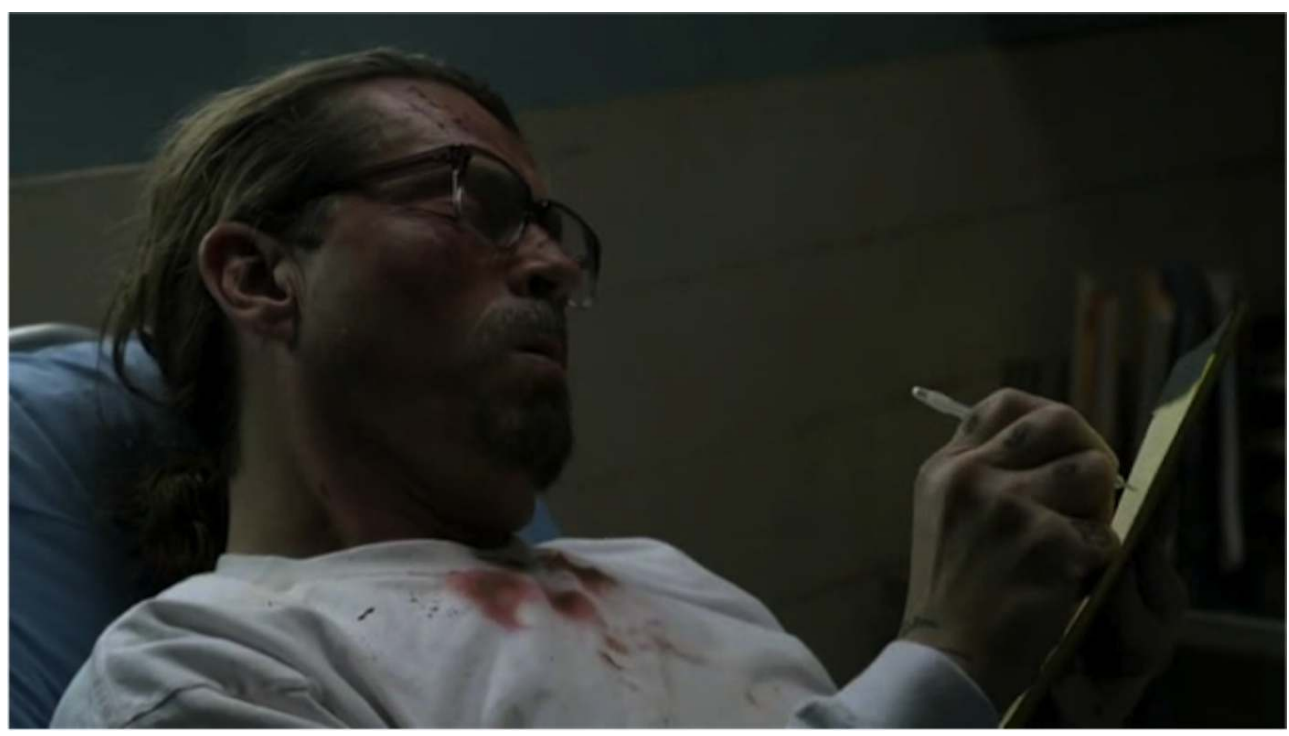


13 Parce qu'il est incarcéré, son rôle est limité à celui d'adjuvant ou d'opposant ponctuel pour les affaires du club, au gré des pressions qu'il subit de la part de ses anciens partenaires ou des autorités, et ses confessions sont l'enjeu de multiples intrigues. C'est Kurt Sutter lui-même qui incarne ce personnage et ce choix de distribution produit une mise en abyme, excédant le simple amusement du cameo ${ }^{27}$ et ouvrant un champ métaphorique de la genèse littéraire grâce à deux éléments notables. En premier lieu, il opte pour le prénom Otto, à l'instar du Prince Otto, héros du roman de Robert Louis Stevenson ${ }^{28}$, considéré comme fondateur du genre ruritanien, déjà mentionné comme une référence littéraire de la série. En second lieu, il réserve à ce personnage un sort des plus violents. Otto Delaney subit, et même s'inflige, de nombreux sévices physiques et moraux, qui semblent être des tentatives pour l'abstraire progressivement de la fiction, comme si le tuer radicalement n'était pas suffisant. Véritable man of Mayhem, gardien des secrets du club, il survit jusqu'à la $6^{e}$ saison, et l'on peut peut-être voir dans ses châtiments qui touchent à ses sens - puisqu'il est devenu aveugle et muet -, une évocation symbolique des pouvoirs d'omniscience et d'omnipotence du scénariste, ou même une réminiscence du devin de Thèbes, Tirésias ${ }^{29}$.

Le nombre et la variété des textes et des références culturelles présents dans Sons of Anarchy mettent donc en évidence les enjeux de la création, qu'elle soit littéraire ou télévisuelle. Si cet inventaire atteste sans nul doute de l'usage du fait littéraire dans une fiction où on ne s'y attendrait pas, il invite néanmoins à s'interroger sur sa réelle finalité, compte tenu de ce qui apparaît comme son retrait progressif à mesure que la série avance. Que valent vraiment ces écrits, et tout particulièrement les écrits intimes quand ils n'aident plus à la narration? Afin de questionner plus spécifiquement leur littérarité, examinons leurs principales fonctions dans l'économie de la série, dans la constitution psychologique des personnages et dans la mise en place des relations actancielles.

\section{Les fonctions de l'écrit intime}

\section{Dialogue intergénérationnel, a father to his son}

15 À la manière du spectre du défunt roi du Danemark qui ne veut et ne peut parler qu'à son fils ${ }^{30}$, les mots de J.T. résonnent et font sens dans la lecture et l'exégèse du fils, nouant ainsi un dialogue intergénérationnel. C'est là leur première fonction. Tombé par hasard entre les mains du fils, le manuscrit du père, jusque là enfoui et oublié dans les affaires du passé, revient à la lumière. Dès l'instant de la découverte, s'installe une situation d'échange d'outre-tombe, entre les survivants et les morts, entre le présent et le passé, comme l'annonce d'ailleurs la dédicace: « For my sons. Thomas, who is already at peace. And Jackson, may he never know the life of chaos ${ }^{31}$. $\gg$ Cette perspective dialogique est nourrie par plusieurs coïncidences, trop heureuses pour être aléatoires, qui accroissent la force conative du message. Coïncidences temporelles tout d'abord. Jax trouve le manuscrit au moment précis où il devient père ${ }^{32}$. Ces circonstances narratives sont opérantes, puisque le trouble de Jax, cultivant un " penchant à la mélancolie ${ }^{33}$ » et devenant père - qui plus est d'un enfant prématuré - répond au trouble de J.T. qui débute son journal à la mort de son premier fils, Thomas. Notons aussi que lors de la scène d'ouverture de la série, Jax trouve un livre pour enfants et se souvient de la comptine The Wheel Goes Round and Round qu'il lira ensuite à Abel. D'emblée, la relation père / fils est liée à la transmission des 
textes et des histoires, et très souvent d'ailleurs, Jax écrit ou lit aux côtés de ses fils ${ }^{34}$ (Fig. 3).

Fig. 3 : Dialogues père / fils par écrits interposés (« Booster », 4.02).

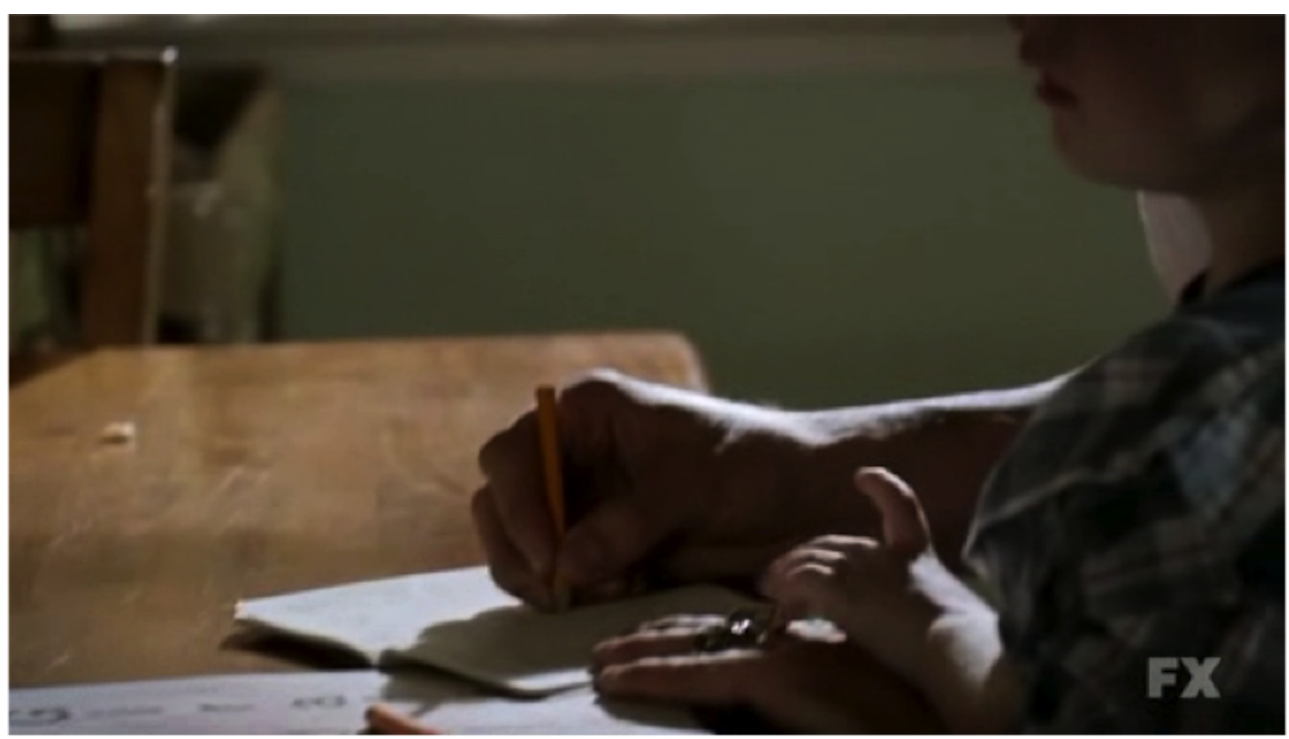

Troublantes coïncidences onomastiques ensuite. Les initiales du père, J.T., valent pour ses deux prénoms, «John » et « Thomas », mais aussi pour ceux de ses trois enfants : JacksonJax, Thomas et (l'illégitime) Trinity. La visée testamentaire du manuscrit n'en est que plus forte. Et il y a bien là un enjeu identitaire fort pour le seul héritier, qui n'a de cesse de répéter dans les premières saisons qu'il n'est pas son père, malgré les ressemblances affirmées par tous. Coïncidence de destination enfin. Désigné dans la dédicace par la $2^{\mathrm{e}}$ personne " you » et le nom «sons», pluriel et singulier, le destinataire est autant le fils, dans la lignée familiale que le "son ", dans la lignée du club. Et c'est bien l'impossible conciliation de ces deux clans qui est au cœur de la progression dramatique, ce qui confère au manuscrit, indépendamment même des actions qu'il suscite, un caractère conflictuel.

\section{Confidence et introspection}

17 Une deuxième fonction cruciale des textes intimes est celle du repli sur soi qui peut prendre la forme de la confidence, lorsque le secret est trop lourd à porter, enjeu majeur des épisodes relatifs aux lettres adultérines. Mais il peut également devenir introspection et impliquer le détachement par rapport à l'action et aux autres. On pense à nouveau à Hamlet et à l'arrivée sur scène du prince en train de lire, qui fait dire à la reine : «But look where sadly the poor wretch comes reading ${ }^{35} »$. En s'adonnant à cette activité solitaire et introspective, Hamlet ne fait que renforcer le sentiment de malaise des autres personnages face à celui qu'ils considèrent comme fou ou pathétique. S'il n'est pas question de santé mentale pour Jax qui se réfugie dans les écrits de son père ou dans sa propre prose, il est toutefois question d'intégrité morale. Aux yeux de la plupart de ses proches, ses échappées dans les textes sont suspectes, car elles diffèrent son action ou la motivent ${ }^{36}$. Habilement d'ailleurs, lors de la lecture ou de l'écriture des textes intimes, la mise en scène insiste sur l'isolement du personnage, face à lui-même, hors du cours continu de l'action. Accomplies dans des lieux d'intimité (chambre, chambre des enfants), 
des lieux de repli et de silence (cimetière) ou encore des lieux en hauteur (toit surplombant le club (Fig. 4) ou capot d'un camion), elles n'en sont que plus propices à la fuite du monde, du groupe et de l'action.

Fig. 4 : Repli sur l'intime (« Seeds », 1.02).

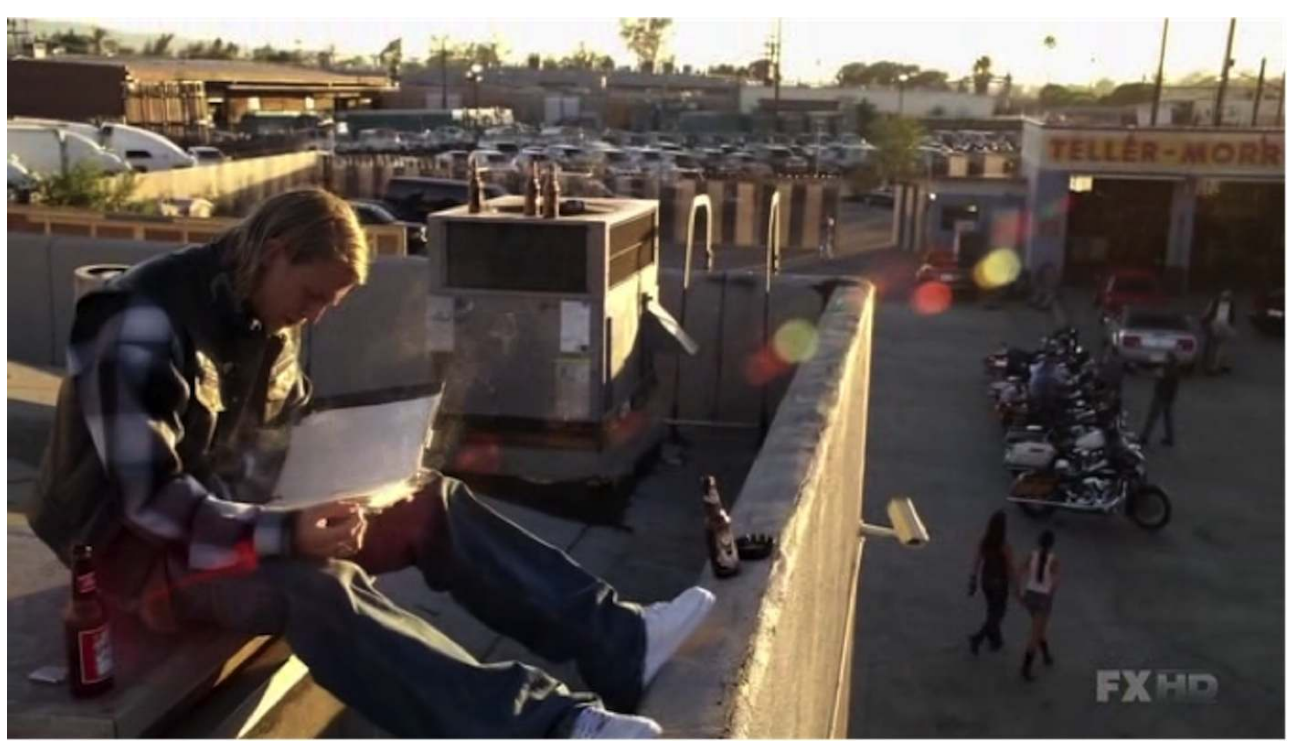

De même, leur mise en voix est signifiante : personne ne lit jamais à voix haute les textes, seules les voix off les transmettent, de telle sorte qu'ils demeurent fantomatiques, dans cette sphère extradiégétique, indices de voix intérieures ou posthumes.

\section{Fantasmer une utopie}

En réponse à Polonius qui l'interroge sur la nature de sa lecture, Hamlet répète «Words, words, words ${ }^{37}$ » et désigne ainsi le prodigieux vertige que les mots provoquent. Les écrits intimes ont cette autre fonction d'être des flots de pensées à interpréter pour ne pas s'y noyer, de textes pour raisonner autant qu'ils résonnent. Marqués par une forte dimension apologétique, ils n'ont de cesse de justifier, légitimer, expliquer, d'autant plus quand cette visée argumentative concorde avec un discours programmatique. Il s'agit alors de plaider pour sa cause en même temps que de plaider pour soi. Et non sans un certain goût pour l'aphorisme, comme on peut le voir dans ces deux exemples empruntés au père: "The true outlaw finds the balance between the passion in his heart and the reason in his mind. The solution is always an equal mix of might and right ${ }^{38}$ » ou au fils : "Children face inward, wallow in their own selfish needs. Men face out, take action on the needs of others ${ }^{39}$. »

Si vérité philosophique il y a, elle est moins détenue par celui qui écrit que par celui qui lit et y associe un sens. Ainsi tournés vers l'élucidation et vers l'avenir de leur lecture, ces textes ne rendent que plus manifeste la difficulté, voire l'impossibilité, de maîtriser le temps présent, lui préférant le fantasme d'un temps futur où les fautes seraient pardonnées, où les actes commis seraient compris et rendus légitimes. En même temps qu'ils sont l'aveu d'une impuissance et d'une faiblesse face aux erreurs du passé et du présent, ces textes empruntent donc à l'utopie et se chargent d'une fonction didactique ouverte sur le lecteur. 


\section{Teller, père et fils}

21 Enfin, il est une dernière fonction à repérer, inférée par le patronyme même des diaristes: "Teller", père et fils, storytellers de père en fils. On pourrait, selon un mécanisme scénaristique bien connu, s'attendre à ce que certains épisodes décrits donnent lieu à des flash-backs et qu'ainsi, les textes rédigés produisent du narratif. Il n'en est rien, pourtant on peut les considérer aussi comme des « histoires ». Et de ce point de vue, en tant que texte intime, ils ne sont pas exempts de la question de leur « vérité ». Du fait que leur visée, au vu des fonctions précédemment analysées, est principalement discursive, leur authenticité n'est toutefois pas un enjeu, personne ne conteste ni leur existence, ni leur signature. Au contraire, c'est bien parce qu'ils sont emplis de la présence et de la force de ceux qui les ont écrits qu'ils deviennent si dangereux, en parfaite résonnance avec le temps présent (et plusieurs personnages payent de leur vie le simple fait de les avoir lus). À tel point que la seule manière de les faire taire, ce n'est pas de les cacher ou de ne pas les lire, mais de les détruire, de les brûler (Fig. 5).

Fig. 5 : Quête et destruction de soi ("Papa's Goods », 7.13).

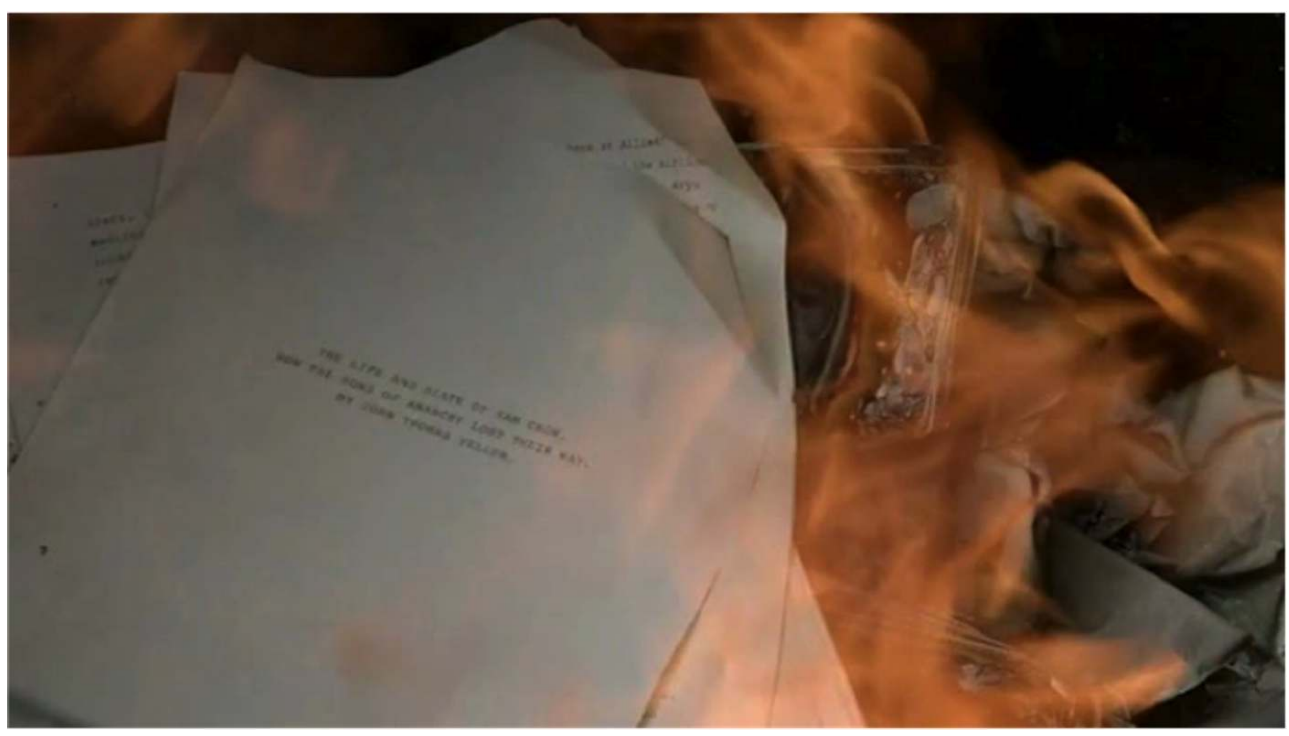

Finalement, bien plus que des « histoires », ils sont comme ces discours séculaires connus et redoutés de tous, c'est-à-dire des mythes, participant pleinement de la légende familiale. Ces textes importent simplement par leur existence, et tous peuvent en parler, sans même les avoir forcément lus.

Cet aspect donne lieu à un retournement final plutôt inattendu, mais rendu possible par le traitement habile des méandres des secrets et des non-dits des textes. Alors que l'exégèse plus ou moins précise du manuscrit et des lettres de J.T. au cours des 4 premières saisons laisse penser qu'il a été tué par le sabotage de sa moto commandité par Clay et Gemma, pour au moins deux motifs - celui de vouloir faire sortir son club du trafic d'armes et celui d'être un mari gênant pour le couple d'amants -, c'est un éclairage nouveau qui est apporté par son vieil ami Jury, confiant qu'il a connaissance du manuscrit mais qu'il ne l'a pas lu - la précision est essentielle - et que tous se sont peut-être trompés ${ }^{40}$. Parce que le mythe a fini par masquer la vérité historique, Jury révèle l'inanité de la thèse du meurtre, puisque J.T., en tant que motard aguerri, aurait forcément senti que sa 
moto avait un problème et que sa mort ne peut être que volontaire. Vérité insoutenable pour Jax, nourri et instruit par l'autre mythologie familiale, qui tue Jury, se condamne par ce crime lui-même à mort et finit par reproduire le geste désespéré - mais libre - de son père.

On le mesure avec cette dernière idée et l'impossibilité à sortir du schéma familial, volonté intime et destin tragique apparaissent comme deux forces autant divergentes que démiurgiques. Tournons-nous à nouveau vers le modèle d'Hamlet afin de mieux exprimer cette tension nodale, au cœur du drame et au cœur de l'être. La question centrale du très célèbre monologue du héros, " être ou ne pas être? ", invite en effet moins à valider un choix entre les deux termes qu'à révéler ce qui sous-tend la prise de conscience de leur opposition. Dans tous les cas, indépendamment de l'issue, que ce soit la vie ou la mort, il s'agit d'exercer avant tout une libre décision.

La première idée que Jax tire du manuscrit est d'ordre très général « kind of hippie shit » et lui semble contraster très fortement avec la réalité du club. Au fur et à mesure des épisodes et de sa lecture, Jax approfondira cette vision. Plus il y réfléchit, plus la figure paternelle lui semble s'éloigner et se dissoudre dans l'incompréhensible. Elle finit par prendre la forme d'un esprit tutélaire, figure mythique et désincarnée du founding father comme en ont connu toutes les sociétés ayant ressenti le besoin de se construire une histoire ${ }^{41}$.

Si le visionnage des trois premières saisons permet à Renaud du Peloux d'expliquer ainsi la déification de la figure tutélaire, il nous semble que la suite de la série va plus loin encore pour révéler les enjeux intimes de ce mouvement d'idéalisation en plaçant justement l'acte d'écrire au cœur de cette question inhérente à la constitution du personnage.

\section{III. Être, écrire et exister}

Les écrits intimes, tant dans leur écriture que dans leur lecture, tant dans leur nonlecture que dans leur non-écriture dans les dernières saisons, rendent manifestes l'énergie qui motive un choix, cette puissance conforme à la vis latine, tout autant force que violence de vie. C'est dans les textes plus encore que dans les actes que s'exerce le rapport conflictuel entre deux sources possibles de cette force : celle appartenant à l'être lui-même ou celle émanant de sa condition d'homme.

\section{Héros au destin tragique}

Dans un ouvrage consacré à sa mise en scène, Pierre Brunel définit sommairement le destin comme :

le déroulement de la vie d'un individu [...] mais ce cours de la vie, au lieu d'être conçu comme libre, peut être senti comme dirigé, mû par une force claire ou obscure, transcendante ou immanente ${ }^{42}$.

En attirant plus l'attention sur ce qui dirige le destin (« une force claire ou obscure, transcendante ou immanente ») que sur sa matière-même, cette formule révèle la duplicité de cette notion, parfois entendue comme la succession des faits d'une vie (le sort, dont on peut faire le récit rétrospectif ou le présage) ou comme ce qui pousse l'individu à agir (une fatalité supérieure ou une motivation personnelle). Dans le cas présent de Sons of Anarchy, la seconde acception réfère au dilemme vécu par le personnage de Jax Teller, héros tragique qui lutte autant pour que contre son destin et qui 
est déchiré entre les actes que lui commande son statut et ceux que lui inspire son caractère. Mais le premier sens de " cours de la vie » importe également pour la conduite d'un récit sériel, puisqu'il désigne finalement l'ensemble des arcs narratifs, depuis un point de départ sur lequel il nous paraît intéressant de s'arrêter. Dans Hamlet, ce qui initie l'action, c'est le devoir de vengeance du fils commandé dès le début de la pièce par le père lui-même ${ }^{43}$ - et c'est l'absence d'action de ce dernier qui génère le tragique shakespearien; au contraire, dans Sons of Anarchy, les représailles abondent bien avant que ne soit fixé chez le héros un impératif de vengeance. Fondus dans une multiplicité d'intrigues secondaires qui ont plus trait aux affaires du club qu'au conflit familial et dans une violence quotidienne tout au long des 7 saisons, les actes perpétrés par Jax n'apparaissent jamais comme immédiatement motivés par le (seul) réel motif, à savoir venger la mort de son père. Hamlet « est presque tout le temps en marge de la vengeance [...], dans la position du metteur en scène ou de l'acteur qui se regarde jouer, spectateur de son propre spectacle ${ }^{44}$ » et, si l'on en croit Nietzsche, il pêche par connaissance, puisqu'

[il n'a] plus que du dégoût pour l'action, [parce que son] action ne peut rien changer à l'essence immuable des choses; [il] trouv[e] ridicule ou avilissant qu'on [lui] demande de réordonner un monde sorti de ses gonds. La connaissance tue l'action, parce que l'action exige qu'on se voile dans l'illusion. Telle est la leçon d'Hamlet ${ }^{45}$.

Jax, quant à lui, semble contenir sa Némésis sous la vaillante lame de la Faucheuse. S'ils étaient un temps tempérés par des discours préliminaires ou analytiques, ses crimes finissent par emplir tout le champ de l'action aux dépends de la réflexion. Proférant dans la $7^{\mathrm{e}}$ saison qu'il n'a plus de vision et qu'il ne voit que ce qui est en face de lui ${ }^{46}$, il plonge dans une violence de laquelle il ne pourra revenir. Le point d'acmé étant le matricide auquel il est conduit. Cet acte irrémédiable est analysé par Nero, figure tutélaire positive, qui interroge Jax sur les conséquences de ce crime, parce qu'il semble plus avisé de l'ampleur des conséquences d'un tel geste, peut-être grâce à son homonyme antique, Néron matricide d'Agrippine ${ }^{47}$. Il prévient donc que ce crime, plus que nul autre, entraînera la destruction de soi, d'autant plus que Jax, contrairement à l'empereur romain déléguant la tâche à ses soldats, sera la main autant que le cerveau.

Avant ce point de non-retour, toutes les actions et décisions du héros n'ont eu de cesse d'osciller entre deux responsabilités : celle du Prince, futur roi, devant assumer à la fois un héritage et une destinée, exerçant une volonté d'être et celle du père et du mari, devant assumer des choix inédits et intimement motivés, exerçant une volonté d'exister. L'analyse de Paul Ricœur des différentes formes du tragique éclaire sur la puissance créatrice et non pas ankylosante de cette tension. Si le tragique est tout à la fois la manifestation du conflit et de l'irrémédiable, il est aussi ce qui pousse le héros à agir, à lutter.

Sans la dialectique du destin et de la liberté, il n'y aurait pas de tragédie; la tragédie demande d'une part une transcendance [...] hostile [...] et d'autre part le surgissement d'une liberté qui retarde l'accomplissement du destin, le fait hésiter et paraître contingent au sommet de la « crise " pour enfin le faire éclater dans un " dénouement ", où son caractère fatal se découvre ultimement; sans le retard de la liberté héroïque, le destin serait comparable à la décharge de la foudre selon l'image de Solon ; la liberté héroïque introduit, au cœur de l'inéluctable, un germe d'incertitude, un délai temporel, grâce à quoi il y a un "drame ", c'est-à-dire une action qui se déroule sous l'apparence d'une destinée incertaine; ainsi retardé par le héros, le destin, implacable en soi, se déploie dans une aventure contingente en nous ; ainsi naît l'action tragique [...]

[T] out est passé, [le spectateur] connaît l'histoire, elle est révolue, elle a eu lieu ; et 
pourtant il attend qu'à travers le fortuit, qu'à travers l'incertitude du futur, la certitude du passé absolu survienne comme un évènement neuf: maintenant le héros est brisé ${ }^{48}$. surgissement d'une liberté qui retarde », «sans le retard de la liberté héroïque », «un délai temporel », « ainsi retardé »), est matérialisé et occupé par les moments consacrés à la lecture et à l'écriture ; les textes intimes sont désignés comme des tentatives d'action pour tempérer le cours tragique, à la manière des interventions du chœur antique. Mais, différence notable, leur portée intime leur confère une dimension supplémentaire, celle de la reconquête d'une identité individuelle, alors qu'il est moins question d'individu que de fonction héroïque dans la tragédie antique. Les textes intimes consistent donc à retrouver qui on est pour tenter d'échapper au fatum. Ils sont chargés d'une "fonction élucidante " grâce à laquelle on peut espérer « rendre cohérente son histoire de vie, à faire entrer son passé dans son présent et à conférer à ce passé - fût-il fait de ruptures, de souffrances, et de bonheur - un sens qui serve pour [l'auteur] et pour le lecteur ${ }^{49}$ ». Même si ces temps de sursis génèrent des échecs, notamment en raison du poids du secret véhiculé par la pression de l'entourage, il est bien question dans l'écriture et la lecture d'exercer temporairement une volonté intime et de devenir en cela héroïque. Jax est bien le seul personnage à accomplir un tel voyage dans la série, à explorer des territoires de l'intime et du tragique où peu sont allés. On pense alors bien évidemment à đEdipe qui traverse par deux fois l'Achéron et qui est aussi coupable de la mort de ses parents - par le parricide commis et le suicide de Jocaste -, mais on pense aussi, pour la force subversive qu'il ne cesse d'affirmer, à un autre modèle littéraire, Prométhée.

\section{Figure prométhéenne, «Entre émancipation et destruction »}

heros de Sons of Anarchy oscille " entre émancipation et destruction » pour reprendre le titre d'un article que François Flahault consacre à l'analyse de l'évolution de la figure prométhéenne ${ }^{50}$. Il partage en effet avec le Titan plusieurs valeurs. Celle du sacrifice de soi, quand son sort individuel est étouffé par les contingences collectives ; celle de la lutte de l'humain contre une force supérieure, puisqu'il répète les gestes de son père ; celle de la liberté de ne pas jouir du confort de sa condition première, parce qu'il remet en cause et finit par nier le modèle de société qui l'a engendré; et enfin, celle de la clairvoyance, mêlée de désir et de renoncement, atteinte au terme son aventure. La pratique de l'écrit et la plongée dans l'intime, en tant que postures introspective et réflexive, font accéder le héros à un statut unique, lui autorisant «[1]a promotion du sujet connaissant (et, par conséquent, la position d'extériorité), le rapport aux choses pris comme modèle de l'action (y compris lorsque celle-ci s'exerce sur les hommes) [et] la référence à un scénario conduisant nécessairement à l'issue escomptée ${ }^{51}$ ». C'est bien cette conscience supérieure qui transparait dans les derniers mots de Jax pour son père, adressés en discours direct à l'endroit où il est décédé. Plutôt que l'écrit, la parole immédiate indique alors que le temps du délai est désormais révolu; à la suite de l'autodafé des textes intimes, l'heure est à la résolution dramatique et à l'apaisement tragique ${ }^{52}$, qui passent par le discours direct.

33 Cette lucidité a déjà émané à l'occasion de quelques extraits intimes lus ou écrits, mais, différence essentielle, elle ne fait plus l'objet d'une quête, elle n'emprunte plus la forme de l'aphorisme, au présent de vérité générale ou au futur, adressé à d'hypothétiques futurs lecteurs - donc des vivants -, comme dans cet exemple : « Know yourselves. Don't be

TV/Series, 12 | 2017 
swayed by fear or history or the opinions of outsiders. Find your own truth. It will lead you to the things you love ${ }^{53}$ ». Désormais acquise, cette vérité est exprimée au passé ou au présent de l'énonciation et s'adresse à un destinataire qui, même mort, est bien avéré.

I think the struggle I understand best, even more than all the things you wanted for SAMCRO, and all we eventually became... The one I feel the most is the war of the mind. Happens when you try to get right with both family and patch. That fear and guilt crippled me. I realized, as I think you did, a good father and a good outlaw can't settle inside the same man. (Pause. Une voiture de police se gare tout près) I'm sorry, JT. It was too late for me. I was already inside it. (Pause. La voiture de police s'approche, bruit du moteur et de la radio) And Gemma... She had plans. It's not too late for my boys. I promise, they will never know this life of chaos. (Pause. La radio de police annonce que le signalement a bien été reçu) I know who you are now. And what you did. (Croassements) I love you, Dad ${ }^{54}$. reste après lui le récit et la parole que Wendy et Nero sont chargés de porter aux enfants, tels des figures d'Horatio ${ }^{56}$, rien dans la mise en scène des dernières instants de la série n'augure le «spectacle » et la solennité de la marche funèbre annoncés par Fortinbras ${ }^{57}$. Alors que les trompettes et les canons résonnent sur la scène au début de la pièce pour la mort du père et à la fin pour la mort du fils, ce sont deux corbeaux picorant sur le bitume qui ouvrent et closent Sons of Anarchy. Bien moins tonitruants que la fanfare shakespearienne, leurs craillements évoquent surtout à notre avis la solitude et le néant. S'opère ainsi un retrait très significatif de tout spectacle - au sens visuel du terme tragique, propice à briser la valse de trahison et de violence et, peut-être même pour les survivants, son souvenir. Sans doute que, dans une série télévisée du Xxi ${ }^{e}$ siècle, il est plus prégnant d'affirmer le conflit intime, dans toute sa finitude, que d'infliger le poids du tragique, comme sur la scène élisabéthaine. Sans doute aussi que si une des finalités de la série est de s'inscrire dans la continuité du message libertaire et anarchique des théories de Proudhon, de Goldman ou de Kropotkine et d'affirmer l'inaptitude du système étatique à promouvoir la liberté individuelle ${ }^{58}$, elle expose aussi, tout au long du parcours initiatique vécu par Jax, à quel point ces choix relèvent d'une fureur de vivre.

Pour conclure, convoquons deux ultimes pistes de lecture pour l'interprétation des textes et du destin tracé par la série. La première doit rendre compte du paradoxe du geste final et fatal de Jax Teller, qui reproduit celui de son père et qui abandonne le club et ses fils, contrairement aux principes qu'il n'aura eu de cesse de répéter ${ }^{59}$. Le destin n'est plus ni une visée prospective - une histoire à écrire - ni un parcours rétrospectif - une histoire à lire -, mais un cycle auquel il est impossible d'échapper. Jax commet donc l'impensable mais avec le soin d'une mise en scène qui l'inscrit dans une révolution parfaite ${ }^{60}$. Entre le père et le fils, pourtant, un acte fondamental diffère : la conservation du texte intime. Rangé avec les certificats de naissance et de mort ${ }^{61}$, le manuscrit du père fait finalement 
figure d'un texte des origines ${ }^{62}$. Et c'est bien à cette question cruciale de la naissance que renvoient les très récurrentes allusions de Gemma, la mère, au sang et à l'ADN, qui justifient finalement tous les actes, même les pires qui soient ${ }^{63}$. Ce choix scénaristique, à l'instar de nombreux autres, témoigne des constantes réappropriations des références shakespeariennes tout au long de la série, ce qu'exprime avec pertinence Sylvaine Bataille :

The series exploits the play as a source of familiar patterns, but also relies on the historically and culturally distant aspect of Shakespeare's work. The echoes from Hamlet emphasize the singularity and the strangeness of the community of bikers and intimate that it belongs to another era, that is a remnant of the past, ruled by political principles and cultural customs coming from another age ${ }^{64}$.

Du texte des origines au texte sacré, il n'y a qu'un pas à franchir pour la seconde hypothèse, qui mène à l'idée d'une transposition biblique, dans laquelle les journaux intimes auraient valeur d'évangiles et les lettres d'épîtres. C'est dans l'utilisation du thème de gospel traditionnel, John the Revelator ${ }^{65}$, que nous trouvons le meilleur indice de cette possible correspondance. Sur le mode de l'antienne, la chanson questionne «Who's that writin ${ }^{66}$ ? " et répète cette réponse : «John The Revelator », désignant l'auteur du livre des "Seven Seals", ou livre de l'Apocalypse. Au moment où il résonne, le manuscrit du père apparaît encore comme un texte contenant les réponses pour l'avenir et légué comme un héritage plein de promesses.

The message is clear; the Truth has now been revealed to Jax (his name is even a kind of Christogram), and it falls to him to do what must be done to restore justice to the club. [...] [ The Sons of Anarchy] were lost, but now with Jax's help and his father's " gospel » they shall be found ${ }^{67}$.

39 Mais malgré ces équivalences, appuyées par d'autres rapprochements onomastiques ou iconographiques ${ }^{68}$, cet ancrage théologique ne nous semble pas le plus important. En effet, cette analyse a voulu montrer que les écrits intimes menaient à la dénégation d'un prédéterminisme, comme l'affirment d'ailleurs ces mots de Jax écrits pour ses enfants : " Every day is a new box, boys. You open it, you take a look at what's inside. You're the one who determines if it's a gift or a coffin ${ }^{69}$. 》

La même chose est à dire de l'écrit intime : cadeau, exhumé tel un trésor, ou cercueil, " temple de la mort élevé à la clarté [des] souvenirs ${ }^{70}$ ", pour citer Chateaubriand et ses Mémoires d'outre-tombe. C'est par cette ambivalence que les textes intimes accèdent à une dimension littéraire, celle consignée par les théories de la réception, conférée par leur destination, par l'acte de lecture qui leur donnera sens, un sens ou des sens. Et ce jusqu'aux derniers mots que l'écran affiche - l'extrait de la lettre d'Hamlet à Ophélie - qui invitent les téléspectateurs autant à la césure qu'au souvenir.

\section{BIBLIOGRAPHIE}




\section{Sur Hamlet}

SUHAMY Henri (dir.), Hamlet, William Shakespeare, Paris, Ellipses, 1998.

SUHAMY Henri (dir.), Dictionnaire Shakespeare, Paris, Ellipses, 2005.

SUHAMY Henri, Hamlet, Lear, Macbeth, Histoire de personnages shakespeariens, Paris, Ellipses, 2010.

SHAKESPEARE William, The Tragedy of Hamlet, Prince of Denmark, [1603], Texte original et version française par André Lorant, Paris, Aubier, 1998.

\section{Sur Sons of Anarchy}

BATAILLE Sylvain, « "Hamlet on Harleys": Sons of Anarchy's appropriation of Hamlet », Shakespeare On Screen: Hamlet, éd. Sarah Hatchuel et Nathalie Vienne-Guerrin, Publications des Universités de Rouen et du Havre, 2011, p. 329-344.

DUNN George A., Jason T. EBERL, William IRWIN (dir.), Sons of Anarchy and Philosophy, Brains before Bullets, John Wiley \& Sons, 2013

DU PELOUX Renaud, Sons of Anarchy, La Guerre perpétuelle, Paris, PUF, 2013

DE SAINT-MAURICE Thibaut, « L'Anarchie selon le SAMCRO, est-ce l'ordre ou le chaos? », Philosophie en séries, Saison 2, Paris, Ellipses, 2011, p. 119-130.

\section{Sur l'écriture de l'intime}

BOGAERT Catherine, Philippe Lejeune, Le Journal intime, Histoire et anthologie, Paris, Les éditions Textuel, 2006.

GIRARD Alain, Le Journal intime, Paris, Bibliothèque de Philosophie Contemporaine, PUF, 1963.

STRASSER Anne, «Quand le récit de soi révèle la fonction élucidante de l'écriture », Temporalités, $\mathrm{n}^{\circ}$ 17, 2013. URL : <http://temporalites.revues.org/2419>

\section{Sur le destin}

BRUNEL Pierre (dir.), La Mise en scène du destin, Paris, Didier Érudition, CNED, 1997.

FLAHAULT François, « Entre émancipation et destruction ", L'Idéal prométhéen, Communications, $\mathrm{n}^{\circ}$ 78, 2005. p. 5-49. URL : </web/revues/home/prescript/article/

comm_0588-8018_2005_num_78_1_2272>.

RICOEUR Paul, Philosophie de la volonté 2, Finitude et culpabilité, Paris, Aubier, 1988.

\section{ANNEXES}


Annexe - Tableau récapitulatif des apparitions des textes intimes

\begin{tabular}{|l|c|c|c|c|c|c|c|c|c|c|c|c|c|c|}
\hline & $\begin{array}{c}\text { Ép. } \\
1\end{array}$ & $\begin{array}{c}\text { Ép. } \\
\text { 2 }\end{array}$ & $\begin{array}{c}\text { Ép. } \\
3\end{array}$ & $\begin{array}{c}\text { Ép. } \\
4\end{array}$ & $\begin{array}{c}\text { Ép. } \\
5\end{array}$ & $\begin{array}{c}\text { Ép. } \\
6\end{array}$ & $\begin{array}{c}\text { Ép. } \\
7\end{array}$ & $\begin{array}{c}\text { Ép. } \\
8\end{array}$ & $\begin{array}{c}\text { Ép. } \\
9\end{array}$ & $\begin{array}{c}\text { Ép. } \\
10\end{array}$ & $\begin{array}{c}\text { Ép. } \\
11\end{array}$ & $\begin{array}{c}\text { Ép. } \\
12\end{array}$ & $\begin{array}{c}\text { Ép. } \\
13\end{array}$ & $\begin{array}{c}\text { Ép. } \\
14\end{array}$ \\
\hline Saison 1 & & & & & & & & & & & & & & \\
\hline Saison 2 & & & & & & & & & & & & & & \\
\hline Saison 3 & & & & & & & & & & & & & & \\
\hline Saison 4 & & & & & & & & & & & & & \\
\hline Saison 5 & & & & & & & & & & & & & \\
\hline Saison 6 & & & & & & & & & & & & \\
\hline Saison 7 & & & & & & & & & & & & & \\
\hline
\end{tabular}

$\begin{aligned} & \text { Manuscrit de J.T. Teller } \\ & \text { Lettres de J.T. Teller pour Maureen Ashby } \\ & \text { Lettre de Jax Teller } \\ & \text { Journal de Jax Teller }\end{aligned}$
$\begin{aligned} & \text { Couleur vive : texte lu ou lisible } \\ & \text { Couleur atténuée : texte vu ou évoqué }\end{aligned}$

\section{NOTES}

1. Témoignant des réactions de bikers avec lesquels il s'est lié, Kurt Sutter qualifie ainsi sa série : « For me, the stories are huge on Sons - it's an epic show and it's pulpy. » Pointant que la multiplication des intrigues et la tension dramatique importent plus que la réalité documentaire, il prête même cette phrase à un biker devenu spectateur: "It's a soap opera but it's our soap opera. » Steven Kurutz, "Sons of Anarchy Creator Kurt Sutter On the Cult Biker Show », The Wall Street Journal, 30 novembre 2009. Ajoutons que par ailleurs Sutter produit et présente une série documentaire de six épisodes sur les hors-la-loi aux États-Unis, Outlaw Empires, Discovery Channel, 2012, dont le deuxième épisode est consacré aux bikers.

2. Pour une étude de cette promotion affichée, voir Sylvain Bataille, «"Hamlet on Harleys": Sons of Anarchy's appropriation of Hamlet ", Shakespeare On Screen: Hamlet, éd. Sarah Hatchuel et Nathalie Vienne-Guerrin, Publications des Universités de Rouen et du Havre, 2011, p. 329-344.

3. Ibid., p. 329.

4. "Doubt thou the stars are fire, / Doubt that the sun doth move, / Doubt truth to be a liar, / But never doubt I love. ", William Shakespeare, The Tragedy of Hamlet, Prince of Denmark, [1603], Texte original et version française par André Lorant, Paris, Aubier, 1998, acte II, scène 2, v. 116-119, p. 156.

5. La découverte de ce secret et de cette famille cachée est au cœur de l'intrigue de la $3^{\mathrm{e}}$ saison, dont une grande partie de l'action est déplacée en Irlande.

6. C'est parce que l'enfant a utilisé une arme revendue par les Sons of Anarchy que l'étau juridique et moral se resserre sur la bande de Jax Teller. Acculé de toutes parts et voulant préserver son organisation, le héros ordonne le meurtre de la mère du jeune garçon («One One Six », 6.02). Ce geste doublé de l'exécution de sang froid de la mère de Venus, Alice Noone, (« Sweet and Vaded ", 6.07) ouvre une funeste série de matricides, dont le point d'acmé est celui de sa propre mère («Red Rose », 7.12). Rappelons que l'accident mortel de J.T Teller, son père, coûte également la vie à une mère de famille, Emily Putner.

7. Alain Girard, Le Journal intime, Paris, Bibliothèque de Philosophie Contemporaine, PUF, 1963, p. 522.

8. Philippe Lejeune, Catherine Bogaert, Le Journal intime, Histoire et anthologie, Paris, Les éditions Textuel, 2006, p. 142. 
9. Précisons que, si nous soulignerons certains rapprochements à propos, notre étude ne porte pas spécifiquement sur les enjeux d'une adaptation de la pièce de Shakespeare.

10. Cf. par exemple « A Mother's Work », 6.13.

11. Gemma Teller est l'un des rares personnages à lire la Bible («Fix», 2.03), mais elle est aussi celle qui l'utilise à des fins moins morales («Pilot », 1.01).

12. Upton Sinclair, The Jungle, New York, Doubleday, 1906. Ce roman est lu par Tara Knowles (« Fix », 2.03).

13. Edgar Rice Burroughs, The Mad King, Chicago, McClurg, 1926. L'ouvrage donne son nom à l'épisode dans lequel il apparaît, arrivé aux mains de Clay Morrow lors de son séjour en prison (« The Mad King ", 6.05).

14. Charlotte, Emily et Anne Brontë, Poems by Currer, Ellis, and Acton Bell, London, Londres, Aylott and Jones, 1846. Les lectures se font entendre lors des épisodes "Faith And Despondency ", 7.10 et «Red Rose », 7.12.

15. " Family Recipe », 4.08.

16. Héros de la série The Walking Dead (AMC, 2010-).

17. "Caregiver", 3.03.

18. «Playing with Monsters ", 7.03.

19. Sur cette question, cf. Charlene Elsby, «My Skin, My Self, SAMCRO's Ink and Personal Identity », in Sons of Anarchy and Philosophy, Brains before Bullets, George A. Dunn, Jason T. Eberl, William Irwin (dir.), John Wiley \& Sons, 2013 [p. 107-113]. Grâce à ces écrits rituels, le corps devient texte, ce qui n'est pas sans rappeler le parchemin médiéval et une thématique centrale de la série suivante de Kurt Sutter, The Bastard Executioner (FX, 2015). Ajoutons aussi que les écussons arborés sur les gilets de cuir, les patches, sont des manières de signaler en toutes lettres le rang de chacun des membres du gang («President », «V. President », « First 9 », « Sgt at Arms », « Prospect », etc.), bien plus explicitement que les galons militaires.

20. Les deux groupes, parce qu'ils sont institués des deux côtés d'une frontière éthique infranchissable, ne peuvent trouver d'entente. Tous ceux qui s'essaient à des négociations d'un côté ou de l'autre échouent et meurent, Jax y compris. Les dernières paroles de Chibs au sheriff Althea Jarry n'en sont qu'une nouvelle affirmation : "From now on, I'm just a cop, you're just an outlaw. We both do what we have to do. » («Papa's Goods », 7.13).

21. "The Revelator ", 1.13.

22. "Sovereign », 5.01.

23. "The Revelator", 1.13 .

24. «Booster ", 4.02 .

25. «Una venta », 4.04.

26. Roland Barthes, «Écrivains et écrivants », Essais critiques, Paris, Seuil, (1964), 1981, p. 150.

27. Jeu d'apparition du réalisateur dans son film, tel Alfred Hitchock, ou du créateur dans sa série, tel Aaron Sorkin.

28. Robert Louis Stevenson, Prince Otto, Londres, Chatto \& Windus, 1885.

29. La référence à đdipe est également possible, mais elle convient mieux au personnage de Jax Teller, qui est inscrit dans une plus grande dynastie, assimilable à celle des Labdacides.

30. HAMLET. Did you not speak to it ?

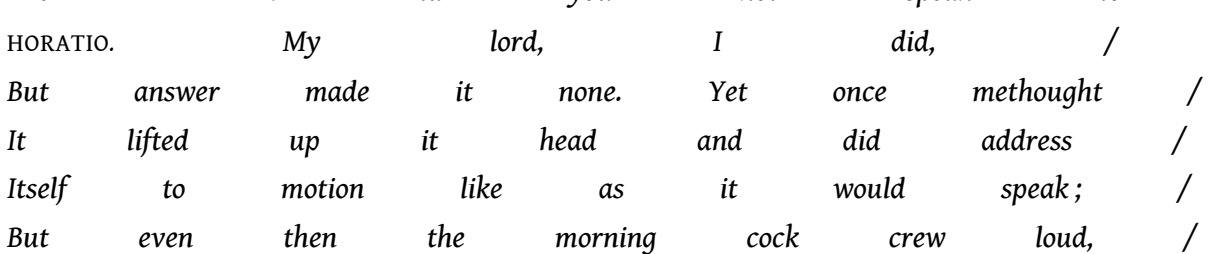

And at the sound it shrunk in haste away / And vanish'd from our sight., William Shakespeare, op. cit., acte I, scène 2, v. 214-220, p. 114. 
31. «Pilot», 1.01 .

32. Gemma, sa mère, sachant ce qu'elle risque à la lecture de ces confessions, ne manque d'ailleurs pas de souligner cette coïncidence à Clay Morrow, son nouveau mari, tout aussi coupable, "The Revelator ", 1.13.

33. Renaud du Peloux, Sons of Anarchy, La Guerre perpétuelle, Paris, PUF, 2013, p. 24.

34. À cet égard, contrairement à ce que semble croire Jax, la destruction des textes à la fin de la série ne pourra rompre ce cycle, puisque les «survivants", Nero et Wendy, sont investis de la mission de dire aux garçons orphelins de père qui était celui-ci (JAX. When the time comes, she needs to tell my sons who I really am. "Papa's Goods », 7.13). Passée dans le logos, l'histoire auparavant écrite deviendra légende.

35. Op. cit., acte II, scène 2, v. 168, p. 158.

36. Ceci est surtout vrai dans les premières saisons, où l'on repère que deux décisions difficiles (bannir un membre, "Patch Over", 1.04 et devenir nomade, "Balm», 2.10) sont d'abord temporisées par la lecture du manuscrit et ensuite motivées par son exégèse. Les téléspectateurs - autant que le héros - trouvent matière à réflexion dans ces échos entre l'écrit et le geste.

37. Op. cit., acte II, scène 2, v. 191, p. 160.

38. « Fun Town», 1.03.

39. «Darthy», 5.12.

40. "The Separation of the Crows", 7.08.

41. Du Peloux, p. 25.

42. Pierre Brunel, «Introduction » in La Mise en scène du destin, sous la dir. de Pierre Brunel, Paris, Didier Érudition, CNED, 1997, p. 8 [p. 7-12].

43. "So art thou to revenge, when thou shalt hear. ", William Shakespeare, op. cit., acte I, scène 5, v. 7, p. 130 ; «Revenge his foul and most unnatural murder. », ibid., v. 25, p. 132.

44. Jean-Pierre Villquin, «Hamlet, tragédie de la vengeance?", in Hamlet, William Shakespeare, Henri Suhamy (dir.), Ellipses, 1996, p. 17.

45. Friedrich Nietzsche, La Naissance de la tragédie, Fragments posthumes, Automne 1869 Printemps 1872, Euvres philosophiques complètes, I, Textes traduits par Michel Haar, Paris, NRF, Gallimard, 1977, p. 69-70.

46. JAX. I don't have a vision anymore. All I see is what's right in front of me. «Toil and Till ", 7.01.

47. NERO. But a son killing his mother? Jax, that's a wound that's too deep to heal. And I know you, Mano. That's gonna swallow you up. That's gonna destroy you, man. "Suits of Woe », 7.11. Il y a dans ce seul personnage de Nero Padilla de multiples résonnances antiques. En effet, le parallèle ici présenté peut encore être nourri par le fait que le fils de Nero s'appelle Lucius, à l'identique du nom de naissance de l'empereur romain (Lucius Domitius Ahenobarbus). Une fois adopté par Claude, quatrième empereur de Rome et nouveau mari de sa mère Agrippine, il devient Néron et grandit donc, comme Jax Teller avec Clay Morrow ou Nero Padilla, au contact d'un père de substitution.

48. Paul Ricoeur, "Le dieu méchant et la vision "tragique" de l'existence », Philosophie de la volonté 2, Finitude et culpabilité, Paris, Aubier, 1988, p. 363-364.

49. Anne Strasser, «Quand le récit de soi révèle la fonction élucidante de l'écriture », Temporalités , n¹7, 2013. URL : <http://temporalites.revues.org/2419>. Consulté le 29 janvier 2016.

50. François Flahault, « Entre émancipation et destruction », L'Idéal prométhéen, Communications, $\mathrm{n}$ 78, 2005. p. 5-49. URL: </web/revues/home/prescript/article/ comm_0588-8018_2005_num_78_1_2272>. Consulté le 29 janvier 2016.

51. Ibid., p. 33.

52. Notons d'ailleurs que le personnage de Gemma, coupable du meurtre de Tara, se terre dans un monologue dès le début de la $7^{\mathrm{e}}$ saison. Plus que l'indice d'un éventuel trouble mental, ce discours direct nous apparaît comme le marqueur d'une résignation tragique de la mère en attente du châtiment de son fils, telle Clytemnestre tombant sous le bras de son fils Oreste.

53. «Straw», 6.01. 
54. «Papa's Goods ", 7.13. Nous ajoutons les didascalies.

55. JAX. « When the time comes, [Wendy] needs to tell my sons who I really am. I'm not a good man. I'm a criminal and a killer. I need my sons to grow up hating the thought of me. ", " Papa's Goods », 7.13.

56. "Horatio, I am dead, / Thou livest. Report me and my cause aright / To the unsatisfied", Shakespeare, op. cit., Acte V, scène 2, v. 329-331, p. 314 ; « So tell him, with th'occurents more and less / Which have solicited - the rest is silence. ", ibid., v. 348-349, p. 316.

57. «Where is this sight?", ibid., v. 353, p. 316 ; « Let four captains / Bear Hamlet, like a soldier, to the stage, / For he was likely, had he been put on, / To have proved most royally; and, for his passage, / The soldiers' music and the rites of war / Speak loudly for him. ", ibid., v.386-391, p. 318.

58. Cf. à ce propos, Du Peloux, p. 79 et suiv. L'épisode "Na Trioblóidí», 2.13, laisse entendre l'extrait d'un texte politique, insistant sur la profonde humanité de celui qui choisit un mode de vie anarchique : "And if the spectacle [of society] touches him, if it rouses interest and compassion, his feeling will be for those tender ones of the great human flock whom the shepherds shear and starve and sell and do not feed. ». La citation est empruntée à William Francis Barry, "The Progress of Nihilism ", The Dublin Review, vol. 99, 1886, p. 19. Texte disponible en ligne: URL: <https://archive.org/ details/3sdublinreview16londuoft>. Consulté le 29 août 2016. Cf. aussi Thibaut de Saint-Maurice, «L'Anarchie selon le SAMCRO, est-ce l'ordre ou le chaos? », Philosophie en séries, Saison 2, Paris, Ellipses, 2011, [p. 119-130].

59. Lors d'un échange avec Chibs concernant l'état préoccupant de Juice, qui est contraint à coopérer avec la police, Jax profère que le suicide n'est pas une option : «Sons don't kill themselves ». « Call of Duty», 4.11.

60. Les symboles abondent tout au long du dernier épisode pour annoncer la chute : Jax laisse ses bagues (les deux bagues «SO » et «NS » et son alliance de mariage), troque ses baskets blanches contre de vieilles bottes en cuir et échange sa moto contre celle enfin restaurée de son père. Le camion qu'il percute porte le logo « Papa's Goods » (qui donne son nom à l'épisode), figurant au sens propre la volonté de détruire tout l'héritage. Ajoutons que cette révolution est valable sur le plan personnel - puisqu'il « accomplit » son destin -, mais aussi sur le plan politique en ce qui concerne les affaires du club - puisque ses dernières décisions en tant que président permettent de relancer le trafic d'armes mais surtout d'admettre comme membres un noir (T.O. Cross, joué par Michael Beach) et un homosexuel (Tig Tager, joué par Kim Coates), soit de faire preuve d'une tolérance plus conforme à l'esprit communautaire et hippie de son père qu'à celui sectaire et violent de son beau-père Clay Morrow.

61. Jax apprend de Gemma où se trouve l'ultime copie du manuscrit : "It's in the storage locker, with you and your brother's certificates. Death papers. ", " Red Rose », 7.12.

62. On note à ce propos que le thème occupe d'ailleurs une partie de l'intrigue de la création suivante de Kurt Sutter, The Bastard Executioner (FX, 2015). Interrompue au bout d'une seule saison, la série (qui a peu enthousiasmé les téléspectateurs et les critiques) met notamment en scène un couple formé par un ancien templier, Ludwig Von Zettel (joué par Kurt Sutter), et une guérisseuse, Annora of the Alders (joué par Katey Sagal), chargé de protéger de l'Église un livre sacré, The Book of the Nazarene, révélant les origines de Jésus. (ANNORA. That is what the pages reveal. Jesus was just a man, Wilkin. But God spoke through him. Filled his heart with profound love and divine wisdom. He knew that devotion was a singular experience between man and God. A church built to glorify that could only lead to greed and destruction. History has proven that over and over. Countless lives lost in the service of faith. How could a loving God ever wish that on his children? And the gospels? Filled with much of God's truth and much of man's persuasion. Why does the seraphim keep this a secret? The seraphim carries the Nazarene's words on their flesh. In his original text. To be passed on from generation to generation, so it can never be lost. It will be given to man when the truth of the pages can be understood. "Blood and Quiescence / Crau a Chwsg», 1.10). Il y a fort à parier que cet arc narratif, plus dense que ceux de la vengeance et de l'usurpation d'identité qui occupent la majeure partie de la saison, aurait été plus amplement développé si la production avait continué. 
63. «My son loves deep; hates deeper. It's in our genes. Betrayal is unforgivable. » («Huang Wu », 6.10); «It's who we are, sweetheart » (« Red Rose », 7.12).

64. Bataille, op. cit.

65. "The Revelator ", 1.13.

66. Notons dans l'interprétation du chanteur Curtis Stigers un jeu paronymique entre "writin' ", « writer » et « riding », « rider ", amplifiant plus encore l'ambivalence de cette référence.

67. Peter S. Fosl, "Sons of History, How SAMCRO Lost and Found Its Way», Sons of Anarchy and Philosophy, Brains before Bullets, p. 189-200.

68. Voir Du Peloux sur la relecture du conflit entre Abel et Caïn, «Un ramassis de bons à rien et de déshérités ", p. 89-96 et Johanna Robinson sur les indices invitant à considérer Jax comme une figure christique, "Sons of Anarchy Series Finale Rides Off Into the Sunset on a Worn-Out Metaphor ", Vanity Fair, 10 décembre 2014. URL : <http://www.vanityfair.com/hollywood/2014/12/sons-ofanarchy-series-finale-jax-dies>. Consulté le 29 janvier 2016.

69. "Orca Shrugged», 5.05.

70. François-René de Chateaubriand, Mémoires d'outre-tombe, tome I, Livres 1 à 12, Paris, Le Livre de poche, (1848) 2001, p. 15.

\section{RÉSUMÉS}

Bien qu'ultra-violente et basée sur le quotidien de bikers hors-la-loi, la série Sons of Anarchy (FX, 2008-2014) multiplie les références littéraires (William Shakespeare, Edgar Rice Burroughs, Stephen King), mythologique (๔dipe, Prométhée) et bibliques. Oscillant entre l'allusion légère et l'appropriation perspicace, ces mentions troublent les limites entre un espace culturel fictif et un espace culturel réel et sont pour le créateur Kurt Sutter l'occasion de questionner le travail même de l'écriture. Mieux, grâce à la figure du diariste, qui unit par-delà la mort un père et son fils - tous deux rédigeant des carnets intimes -, Sons of Anarchy établit la lecture et l'écriture comme ressources efficientes de son fonctionnement scénaristique. Mais plus encore que des procédés narratifs, ces actes instillent à la série une distance propice autant à l'affirmation d'une volonté intime dans un parcours initiatique - celui suivi par Jax Teller, successivement jeune homme dérouté, prince flamboyant et roi sanguinaire - qu'à l'émergence d'une force tragique celle entrelaçant ses inconciliables conditions de fils, d'époux, de père et de chef de bande. C'est en cela que les écrits intimes dépassent l'écueil de leur caractère fragmentaire et privé pour devenir de véritables moteurs pour la constitution psychologique et ontologique du héros, grâce à plusieurs fonctions majeures (dialogique, introspective, programmatique et narrative).

Despite its ultraviolence and the fact that it is based on the everyday life of outlaws bikers, the TV show Sons of Anarchy (FX, 2008-2014) cites numerous references to literature (William Shakespeare, Edgar Rice Burroughs, Stephen King), mythology (Ædipus, Prometheus) and the Bible. Whether simple hints or insightful appropriations, these mentions blur the limits between a fictional cultural space and a real one in addition to become the opportunity for the creator Kurt Sutter to question the work of writing itself. Moreover, thanks to the diarists, a dead father and his living son, both intimate writers, Sons of Anarchy considers the acts of reading and writing as efficient means for its plot. But, these actions are more than ordinary narrative devices : they instil into the show a relevant gap that leads to assert an intimate will during the initiatory journey of Jax Teller - successively a confuse young man, a brilliant prince and a murderous king 
- and to rise a tragic might - the same that ties his irreconcilable conditions of son, husband, father and leader. This is how the diaries and the intimate writings fill in their discontinuity and secrecy to help the hero psychological and ontological personality, thanks to their main functions (dialogical, introspective, programmatic and narrative).

\section{INDEX}

Keywords : Sons of Anarchy, Sutter Kurt, diary, tragic, literature, mythology

Mots-clés : Sons of Anarchy, Sutter Kurt, journal intime, tragique, littérature, mythologie

\section{AUTEUR}

\section{VANESSA LOUBET-POËTTE}

Vanessa Loubet-Poëtte est docteure en Linguistique française. Membre du CRPHLL (Université de Pau et des Pays de l'Amour), ses recherches portent sur les mécanismes de l'énonciation. Spécialiste de sémiologie de la littérature et du cinéma, elle a publié plusieurs études sur l'adaptation et les échanges intermédiatiques entre le médium textuel et audio-visuel.

Vanessa Loubet-Poëtte holds a PhD in French Linguistics. Membre of the CRPHLL (University of Pau and Pays de l'Adour), her research focuses on the enunciative structures. Specializing in semiotics in litterature and cinéma, she had published studies of adaptation and transferts between text and audiovisual. 